\title{
New Approaches in Cognitive Radios using Evolutionary Algorithms
}

\author{
Miguel Tuberquia, Cesar Hernandez \\ Technological and Engineering Department, Francisco José de Caldas District University, Colombia
}

\begin{tabular}{l}
\hline \hline Article Info \\
\hline Article history: \\
Received Dec 23, 2017 \\
Revised Apr 2, 2018 \\
Accepted Apr 10, 2018 \\
\hline
\end{tabular}

Keyword:

Ad-Hoc network

Artificial intelligence

Cognitive radio

Evolutionary algorithms

Survey

\begin{abstract}
Cognitive radio has claimed a promising technology to exploit the spectrum in an ad hoc network. Due many techniques have become a topic of discussion on cognitive radios, the aim of this paper was developed a contemporary survey of evolutionary algorithms in Cognitive Radio. According to the art state, this work had been collected the essential contributions of cognitive radios with the particularity of base they research in evolutionary algorithms. The main idea was classified the evolutionary algorithms and showed their fundamental approaches. Moreover, this research will be exposed some of the current issues in cognitive radios and how the evolutionary algorithms will have been contributed. Therefore, current technologies have matters presented in optimization, learning, and classification over cognitive radios where evolutionary algorithms can be presented big approaches. With a more comprehensive and systematic understanding of evolutionary algorithms in cognitive radios, more research in this direction may be motivated and refined.
\end{abstract}

Copyright () 2018 Institute of Advanced Engineering and Science. All rights reserved.

\section{Corresponding Author:}

Cesar Hernández,

Department of Technology,

Francisco José de Caldas District University,

Cl. 68D Bis A Sur No 49F - 70, Bogota, Colombia.

Email: cahernandezs@udistrital.edu.co

\section{INTRODUCTION}

Currently, cognitive radio networks have acquired an essential role in the development of communications, it has been demonstrated the use of cognitive radio in different areas of study such as smart grid [1], television [2], wireless communications [3], and the internet of things [4]. The limited available spectrum and the inefficiency using of the spectrum have become one of the current problems in wireless networks. Cognitive radio (CR) technology has been proposed as a solution to these matters [5]. The use and demand of wireless data have recently increased, and it is expected to maintain this trend due to the continuous demand in wireless applications. It is likely that the monthly use of mobile data will increase eight times before 2020 compared to 2015 [6]. A significant research effort has been devoted to improving the use of data and network performance [7], [8]. The growing proliferation of wireless devices has made the $900 \mathrm{MHz}$ and $2.4 \mathrm{GHz}$ bands congested. At the same time, there are several frequency bands, licensed for operators in the range of 400 to $700 \mathrm{MHz}$, which are used sporadically [5]. Many campaigns have been developed demonstrating that the spectrum is being underutilized by authorized users [9]. In cognitive radio, unlicensed users use the spectrum opportunistically with the restriction that when detecting a licensed user, they must leave the band immediately and move to a new one [10], [11].

As defined in [12], a cognitive radio is an intelligent wireless communication system that is aware of its surroundings. CR has used methods to understand the environment and sought for adapting to statistical variations. There have been important challenges that have been attracted attention to improving the quality of service (QoS). The detection of spectral opportunities, the selection of the best available channel, the 
coordination of several users in accessing the channel, and the opportune channel change when a licensed user is detected are some of the relevant issue presented nowadays [13]. Spectrum Sensing has been characterized as one of the most debated functions of CR, since it is necessary to develop intelligent techniques that select the best spectral opportunity reliably and quickly [14].

Artificial intelligence (AI) plays a crucial role solving some of the gaps present in CR [15]. The AI has allowed tools for analysis, development, and implementation to seek the QoS improving. Works like [16], where a comprehensive survey is carried out in technical applications, show it some of the approaches in AI such as machine learning in CR. Although not only machine learning is the area of AI that seeks to solve the problems mentioned above, evolutionary methods have taken an essential role in addressing these issues. This research intends to provide an art state, which allows readers to have a general view of the evolutionary algorithms (EAs) involved in improving the performance of CRNs. Relevant algorithms in evolutionary methods such as ant colony, artificial bee colony, genetic algorithms and among others will be mentioned in this short survey. The most important contribution will be shown the research presented after the year 2015 until the year 2017.

In 2009, the work proposed in [17] focused their research on the partial problems associated with observation and reconfiguration in the domain of cognitive radio, emphasizing how AI can be used as an application. The research shows the state of the art in the AI techniques involved in CR and describes the proposed works in the areas of; Artificial Neural Networks, Hidden Markov Model, Rule-Base system, ogybased systems, case-based system, and metaheuristic algorithms. The authors concluded that CR engine must be designed taking care the exchange between performance and complexity gave by the application, and the training and learning are necessary to achieve acceptable performance.

In 2013, the authors [18] made a summary of evolutionary algorithms, focusing the research on three aspects that they consider relevant; how EAs contribute to exploration and exploitation, when and how exploration and exploitation are controlled, and how it is achieved the balance between exploration and exploitation. The primary intention of the work is to gradually diminish the common misunderstandings and the erroneous beliefs about the exploration and exploitation of AEs. Furthermore, the authors show characteristics in algorithms and applications on exploration and exploitation, moreover, essential objectives in the cognitive networks. The work does not show a direct link regarding communication networks and cognitive radios.

In 2015, the authors of [19], presented a compilation of learning techniques in AI conducted to date in research that involved CRNs. The authors make a description of the tasks required in communication process in CR and the methods of AI and machine learning that have been applied to improve the current process. The work shows the contributions of neural networks, Support vector machine, genetic algorithms, game theory, reinforced learning, fuzzy logic, decision tree, artificial bee colony, Markov models, Casebased reasoning, Entropia and Bayesian approach in cognitive networks. Finally, the research proposes different points of view and different ways of approaching cognitive radio networks from AI point of view.

In 2016, the work proposed in [20] provided a compressive description of genetic algorithms (GAs) and a large number of techniques implemented mainly focused on wireless communications networks. The paper presents an explanation of GAs, their terminology, definitions, types, components, operators, coding and fitness functions to introduce the readers into the scope and limitations of GAs. The authors detail the areas where GAs can be used within wireless networks such as routing, channel allocation, bandwidth allocation, load balancing, location and quality of service (QoS). The work shows some possible combinations of GAs with artificial intelligence techniques such as game theory, reinforced learning, graph theory, fuzzy logic and systems based on quantum theory with applications in wireless networks. Finally, it shows a number of problems that still present gaps in their development and the possible guidelines that guide GAs to solve the problem.

The contribution of this paper in comparison with the works above will be emphasized the EAs and their applications in CRNs. A description of the most used EAs in the framework of CRNS will be made, as well as some current application in EAs. Finally, some research topics are mentioned under present discussions and shortly how evolutionary algorithms could make approaches in the resolution of problems in CRNs.

The remainder of this article will begin with presenting the most relevant concepts about CR structure in Section 2. Section 3 described the biological foundations on which the evolutionary algorithms are based. Section 4 will show some of the current contributions that evolutionary algorithms did to CR. A comparative evaluation of evolutionary algorithms is described in Section 5. Section 6 will show future and ongoing research challenges. Finally, we conclude the article in Section 7. 


\section{COGNITIVE RADIO}

This section will briefly describe the main characteristics of CR, the principal components, the architecture of which a CR is composed, and the processes involving in CR. Thus, a cognitive radio network is defined as a system that allows the environment to be censored and analyze transmission parameters to make decisions in a dynamic time-frequency space. CR, according to the designation and management of resources seeks to improve the use of the electromagnetic radio spectrum[19]. Therefore, a CR is expected to be smart and able to learn from its experience by interacting with the RF environment. Accordingly, the learning process is an indispensable component that can be provided using artificial intelligence and machine learning techniques [19].

The architecture of CR is distributed in two principal groups:

a. Licensed users or primary user (PU) will occupy the first group called the primary network or licensed network. Those who pay for a specific spectral channel and has the priority in accessing the spectrum. The operations of primary users must not be affected by others users.

b. The second group is called a secondary network or unlicensed network occupied by an unauthorized user or second users (SU), which seeks to occupy the free spectral channels and share the band with the authorized users. Therefore, it constitutes a mainly dynamic network of access in the spectrum [21].

The management of the spectrum is implementing by fours steps that cover the process of how users occupy and share the spectrum.

a. Spectrum sensing: It is the process in which the SUs monitoring the available spectrum bands, capture their information and detect gaps in the spectrum.

b. Spectrum decision: Based on spectrum availability and the internal and external allocation policies, SU users can be assigning a channel.

c. Spectrum sharing: Because multiple SUs are attempting to access spectrum, the CR network must play the role of coordinating the allocation of spectral spaces, as well as preventing various users from crashing in parts of the spectrum and overlapping.

d. Spectrum mobility: When a PU requests access the spectrum and the SU is using the same spectrum promptly. The SU must be mobilized in the direction of a hole to protect the transmission of the PU and continue with the transmission [22]. However, once this situation occurs, there is also a performance degradation [23].

There are couple concepts in a cognitive radio network implemented to express the process of opportunistically channel sharing, which are: Cognitive capacity, this refers to sections of the spectrum that are not being used and can be shared without interfering the transmission of licensed users. The void fractions are denominated in the literature as spectral holes or white spaces [12]. The next concept is the reconfiguration, which is the ability to transmit and receive in a CR. It is programmed to be implemented at multiple frequencies with the use of software technologies [24].

It is important now show, what methods are being used within the CR architecture to improve the processes of access and share the spectrum implemented by SU. This is where evolutionary algorithms play an important role since they have been used in each of the processes of cognitive radio; spectrum detection [25]-[27], Spectrum Decision [28], [29], spectrum division [30] and spectrum mobility [31], [32].

Once the cognitive radio technologies are used in communication networks, calculations must have been made in real time and the processes of detection, decision, sharing, and mobility, must be executed in fractions of a second. Therefore, structures that do not require high computational complexity should be implemented. EA promises to be a tool to achieve in CRNs, since present computational simplicity, reliability, and robust performance when performing their instructions. EAs have also been presented as a solution to problems of optimization, learning, and search. This work is going to present some EAs that have been presented in artificial intelligence as powerful tools for parameters estimating in both classification and prediction. Furthermore, it is important to mention how they work and what algorithms present in state of the art have been used to improve the performance of $\mathrm{CR}$.

\section{EVOLUTIONARY ALGORITHMS}

EAs are a set of machine learning techniques that seek to imitate the robustness of the procedures and structures that biological organisms have used for adaptation and evolution learning [33]. We are going to describe in brief, the technical and fundamental operations by which the evolutionary algorithms have been motivated. The intention is to show the reader a notion of the algorithms and how they can be implemented in the CRNs problems. 


\subsection{Genetics algorithms (GA)}

The GA Algorithm is an EAs technique which is based on methods of inheritance and natural selection inspired by the biology of evolution [15]. The GAs find their utilities on genetic operators of random mutation and recombination through cross-breeding to improve their solutions. The GAs encode their potential solutions in a simple chain of chromosomes as a data structure. Then, they select the best options to apply recombination operators and preserving the desired information [33]. The implementation of genetic algorithms starts with a random population of chromosomes, which evaluate the problem and seek opportunities for reproduction. The purpose is to preserve chromosomes and give the chance of reproducing chromosomes with better solutions. Besides, the operators mentioned above, the genetic algorithms include other essential components such as initial population, genetic representation, fitness function, and mechanism for selection [20].

\subsection{Artificial bee colony (ABC)}

The $\mathrm{ABC}$ algorithm are used to solve multidimensional and multimodal optimization problems. The basic model of a honey bee colony is characterized in two important stages: the nectar collection and the abandonment of the food source. We can characterize three essential components in a honey bee colony; the food source, the employed bees and unemployed bees [34]. Different factors distinguish as a good or bad food source; the most important is the profitability of the food stands out since it indicates how much is left after the extraction begins. The profitability of the food can be represented as a single quantity [35]. The Employed bees associated with a particular food source carry the information about such as the profitability of the source, the distance, and direction concerning to the hive. The employed bees share this information with the unemployed bees with a probability [34].

Unemployed bees are in constant search of food sources to be exploited. These bees are divided into two types; Scouts and Observers. The scout ones look in the surrenders around the hive for food and the looker's ones wait in the hive and establish a source of food through the information shared by the employed bees. The exchange of information through bees is the most crucial event in the formation of collective knowledge. A dance called waggle provides information about the quality of food sources. Once the looker bees observe the waggle, they will analyze and choose the most profitable source to use it as the best source of food [34].

The ABC simulates the behavior of the bees and divides the bees into two groups: one half will be employed bees and the second half will be lookers unemployed bees. For instance, each food source there will be only one employed bee. Therefore, the number of employed bees will be equal to the number of food sources around the hive. The employed bees whose source of food is finished will become an unemployed scout bee.

In brief, the $\mathrm{ABC}$ algorithm is then divided into three steps. First, moving the working bees into the food sources and determining the amount of food. Second, send looker bees to the food source and determine the amount of food. Third, stop the exploitation of food by employed bees when the food is exhausted and convert the bee into a scout to discover new sources of food randomly [34].

\subsection{Ant colony system (ACS)}

Modern AIs technologies have been studied the behavior of ant colony systems. Ant colonies have the characteristic of seeking short routes of food sources and the return way to the nest. Ants lead their way of communicating with each other using the pheromone trails finding the road to follow the closest ant [36]. Therefore, the more ants follow a path, the higher the amount of pheromone deposited on the trail. Mathematically speaking, the probability that an ant will select a path increases with the number of ants that have traveled the path.

In this way the collective behavior of the ants is characterized as a positive feedback process, in other words, it is a process that reinforces itself allowing a very fast convergence as long as there is no limitation in the environment that leads to the exploitation of the solution [37]. Each ant is identified as an agent that leaves a signal that allows making changes in future decisions. Therefore, the set of ants does not converge to a single solution instead they are made a subspace of solutions to then select the best.

Algorithms such as ant colony optimization (ACO) are based on stochastic processes, and social the behavior of the ants described above, introduce solutions to a complex optimization problem [33]. Characteristics such as parallel computing, self-organization, and positive feedback are inherent in ACO. It allows multi-agent optimization to reach global solutions, reduce computing time and computational complexity [38]. 


\subsection{Cuckoo search (CS)}

This method of EA is base on the reproductive behavior of the cuckoo bird species and the way in which they hatch their eggs. The tactic assumed by the birds is to deposit the eggs in a communal nest and remove some eggs that belong to the nest [39]. The eggs deposited by the cuckoo are called alien eggs, and the eggs that are previously in the nests are called host eggs. If the host bird finds parasitic eggs in its nest, it will proceed to get rid of the egg or leave the nest.

Different species of cuckoo have evolved to camouflage their eggs into the host's, or once the alien egg is deposited, it will try to blend and unnoticed by the host bird. The incubation time for the parasitic eggs is less than the host eggs, as a consequence once the cuckoo chick has hatched its first instinct will be to dislodge the host eggs to increase the chance of being fed by the host bird. All these tactics have been developed and evolved to increase the probability that the parasite egg will be breed and fed [39].

In a computational plane, the CS synthesizes the behavior of the cuckoo bird in three rules: First, each cuckoo will leave only one egg when it arrives at a random nest. Second, the best nest with the highest quality of parasite eggs will be taken in the following generations. Third, The number of available host nests can be modified and the probability that a parasite egg will be discovered will be given by a rational $\mathrm{n}$ umber [40].

Each egg in a nest represents a solution new solution. The objective then is to use the new and potentially solutions (alien egg) to replace the previous solutions, which do not represent good solutions according to the problem. The generation of new solutions, new cuckoo eggs, will be implemented through the random route of Lévy flights. The uses of Lévy flights are essential in the implementation since they represent a more efficient the exploration of the bird to find a nest. The length of the steps is longer and is represented by a Lévy distribution [40].

\subsection{Particle swarm optimization (PSO)}

This EA intends to establish and improve communication channels based on the simulation of swarms [33]. Its main application is in the optimization of nonlinear functions [41]. The algorithm seeks to model and understand the basic rules of collective movement and communal regrouping of swarm members. Examples of this behavior are exhibited by different species of animals such as birds, fishes, bacteria, and insects [42]. These rules are based on an information exchange and the physical conservation of space occupied by each member in the swarm at the moment their travel.

In a flocking, for example, some members of the swarm are attracted where the place the food is found. Therefore, the rest of flock eventually are attracted by a dynamic force. This selective group of members that have been attracted firstly is known as the optimum in a search space [41]. Furthermore, the PSO generates a population of random agents in position and speed which represent the entities that make up the swarm. In fact, the random component is added to avoid a consistent pattern and avoid a rigid behavior in the swarm. In each iterative cycle, each agent determines its new velocity concerning the position of the nearest neighbor agent.

Once, some agents have found a solution they will address to it, and according to the information sharing in the swarm, they will fly over a solution space. Each agent that has found a solution will direct its neighboring agents in that region. Indeed, the swarm can reach a reasonable solution thanks to the direction of the first agent. Moreover, each agent is considered as a data structure that contains relevant information about the solution of the optimization problem and is treated in the context as a particle.

The information about the best solution found so far is contained in a particle that indicates the current location in the optimization plane. The subset of agents is seen as neighbors. In short, there are several tasks executed in a PSO at the time by specialized agents. That featured supported the division of labor, improved the speeds up, and performance in comparison to the performance of sequential tasks made it by unskilled workers [34].

\section{EVOLUTIONARY ALGORITHMS CONTRIBUTIONS IN COGNITIVE RADIO}

In this section, we will present the most recent contributions in the literature where evolutionary algorithms play a vital achievement improving the CRNs performance.

\subsection{GA}

In [55] proposed a spectrum allocation model that can be used in named underlay networks (UCSGC). The approach is implemented based on the mathematical model of color-sensitive graph coloring (CSGC). CSGC is deploying for spectrum allocation developments and it has been used in overlay networks. In order to optimize the benefits of the network, the Optimize Genetic Algorithm (OGA) is implemented to allow convergence and avoid remembering the local optimum, in comparison to the traditional GA by 
randomly recruiting individuals in each iteration. The OGA in each iteration selects good fitness from the father and the offspring. Then adds new individuals to produce new parents. This will allow the algorithm to converge to an optimal global with a probability of 1 . The authors make a comparison between the algorithms UCSGC and CSGC, showing the use of UCSGC has greater network benefits. Then compared the algorithms OGA and GA, revealing the OGA algorithm has higher performance.

\section{2. $\mathrm{ABC}$}

In [43], an $\mathrm{ABC}$ algorithm is presented to designate receptors retransmissions using a suboptimal indicator detection of indicators in a binary form. The looker's bees make a greedy selection on employed bees using a probability function allowing more efficient results concerning to typical greedy selection. The improvement presented in this algorithm lies in send scout bees towards all abandoned sources instead of selecting one food source to leave and sending explorer bees to find new food sources. The results are compared with some other optimizations algorithms such as Exhaustive Search Algorithm, Exhaustive Search Algorithm 1-to-1, ACO, Estimation of Distribution Algorithm and Biogeographt-base Optimization. The channel capacity over receivers in a shared bandwidth showing increases with the number of users, as well as an increase in the capacity with the number of retransmissions. These approximations are taking care of PU interference restrictions. In summary, the algorithm presented has a performance close to ESA, which offers an optimal solution, but with a lower computational complexity.

In [44], the authors proposed a solution for the assignment in CR channels using a bee algorithm. The model was formulated base on [45] approximations. The previous work proposed three functions for an optimal assignment of an open-spectrum channel in a CRN. The contributors add a new function for a better decision making. The functions are exposed regarding single-hop flow and described how the channel allocation in CR systems works.

a. Max-sum of reward, (MSR): describes the total use of the spectrum in the system independent of the judgment made in each reward of SUs.

b. Max-min reward (MMR): It is the sum of the users with less allocation in the spectrum

c. Max-Proportional-Fair (MPF): Add a proportional change in the reward for each possible assignment and its own reward.

To decreasing the search space, a mapping process is proposed between the channel assignment and the bee positions. Due to the automatic fingerprints left by the PUs in the spectrum, either by broadcast operation indicators or by access to the database centers, the SUs are able to detect these signals given not to interfere with the operation of the PUs.The function added to increases the judgment for each channel assigned through the SU was; Maximum Contractor Total Reward (MCTR): To take care the limits for the rewards of Non-Licensed Users. The algorithms ABC and bee swarm optimization (BSO) algorithms were formulated using the functions above and show the network performance improves by reducing interference between SUs. The results of this research are compared with the algorithms GA, QGA, CSGC and PSO. Where ABC and BSO perform much better than GA, QGA and CSGC. The ABC and PSO algorithms have a similar performance and the BSO algorithm shows superior performance.

\section{3. $\mathrm{ACS}$}

The work exhibited in [46], a channel hopping algorithm is presented for the selection of a control channel in a heterogeneous, spatial and time-varying spectrum for an Ad-hoc CRNs. The Swarm Aided Stay Jump (SASJ) algorithm is based on ants colony system for the selection of a control channel without the existence of pre-established routes such as access points or base stations. Some characteristics are designated regarding road quality. The real pheromone is searching for the best channel hopping sequence (CHS) offered by the network. An ant system will seek and reinforce the channel with better characteristics between multiple options offered by the network. The algorithm improves the convergence times occurring when each node of an SU seeks to communicate with another node. It is relevant to mention that the way to establish a connection is using a shared channel. The results of this development are simulated in an urban cellular radio environment with constructions hindering direct communication. As a result, the SASJ shows better performance than Rendezvous times (TTR), and expected Rendezvous times (ETTR). The proposed technique gives better results in channels re-selection where there are traffic scenarios or security attacks in contrast to the technique of selecting single channels.

The work proposed in [52], an improved ACO is introduced to increase the spectrum performance in a CRNs. The proposed algorithm quickly converges to an optimal solution due to the reinforced learning which is provided to an accumulated pheromone. The authors represent the number of available channels and unlicensed users in a matrix. The matrix will judge whether a node is available or satisfies the interference restrictions. If the node meets the interference restrictions, the channel will be assigned to the SU together with a benefit. For instance, the SU will be described ant searching for food, in this case, a node. The SU will 
leave a pheromone on the path, and the next SU will select the path with the highest probability of finding a food/node. The algorithm attaches to the traditional ant colony method, the way in which the pheromone spreads its fingerprint, to increase the global search capacity. The pheromones parameters are configured by adjusting the attenuation coefficient, and the path to be followed is limited to specific intervals given by experimentation. The alters above seeking to prevent premature convergence. The ant configuration plays a vital role in the ability to find optimal solutions. If it is selected a small value, the ability to find good solutions will be low, and the benefits will be few. As the number of ants is increased the search capacity for optimal solutions improves and the benefits increase in the same way.It is concluded from the scenarios proposed by the authors that convergence for an optimal solution was achieved by making dynamic changes in the updates of the pheromone as well as the attenuation coefficients.

\section{4. $\mathrm{CS}$}

The development in [47], use the algorithm Improved Cuckoo Search (ICS) to sensing the spectrum in a CR of scarce satellites. The complexity of this network is lower in comparison to cellular telephony networks. Therefore, the search in the shared spectrum is slower for satellite cognitive networks. One of the primary objectives of the ICS concerning the CS is the adjustment of the search parameters (Alpha) and (Pa). The values of Alpha and $\mathrm{Pa}$ decrease with the number of iterations amounts, searching in this way for a faster and more efficient global search. The results obtained were: The probability of discovering a free subchannel indicates a compensation between the sub-channel bandwidth and the sensing time. The convergence rate of an idle band can be reduced with the increases of subchannels to obtain a better stopping speed. The search convergence rate of optimal global outcome in a CR module is carried one search allowing a timesaving in the reach of idle channels. In Short, the ICS dynamically adjusts the space distribution along with the change/discovery probability and looking for the optimization in unoccupied channels capture to avoid redundancy.

\subsection{PSO}

The work proposed in [48], a PSO was implemented to minimize the total service time in a handoff spectrum. It is knowing that the prediction of PU arrivals must be before the spectrum handoff to continue with the transmission in a free channel. Then, a hybrid spectrum model is chosen to take advantage of the spectrum proactive (rapid response) and spectrum reactive (precision in the selection of the target). In fact, a fast spectrum handoff with a very low latency during the spectrum mobility is got it.The authors characterized the communication channel behavior in a busy CRN and implemented a model network with queues M/G/1. Where the arrival time of the users is Markovian, the time service has a general distribution and a single dispatch server. That is, the minimized objective function is defined as the total time service for unauthorized users base on queuing theory and some restrictions. The results show a $25 \%$ reduction in total service time in comparison to a randomized scheme and a $35 \%$ reduction in total service time in contrast to handoff spectrum schemes. As consequently, the presented algorithm reduces the delays caused by handoff.

\section{COMPARATIVE EVALUATION OF IMPORTANT EAs}

In Section 3, the biological models of EAs were presented. Furthermore, the reader can have got a basic idea of how they work and what characteristics the problem should satisfy. Moreover, Table 1 is going to show the computational consideration of the EAs mentioned above as well as the papers in which the algorithms had been implemented in CRNs. Indeed, the reader will be able to visualize more clearly the advantages and disadvantages of the EAs in the current CR literature. 
Table 1. Comparison of Relevant Evolutionary Algorithms

\begin{tabular}{|c|c|c|c|}
\hline EA & BENEFITS & WEAKNESS & $\mathrm{CR}$ \\
\hline$\overline{\mathrm{ABC}}$ & $\begin{array}{l}\text { Ability to explore local solutions } \\
\text { Skill in managing objective costs }\end{array}$ & $\begin{array}{l}\text { Requires new fitness tests in each new } \\
\text { parameter } \\
\text { Possibility of relevant loss of information } \\
\text { Requires a fitness function } \\
\text { High number of evaluation functions } \\
\text { Population solutions increase the } \\
\text { computational cost }\end{array}$ & $\begin{array}{c}{[26],[28],} \\
{[31],[43],[44]}\end{array}$ \\
\hline ACS & $\begin{array}{l}\text { Inherent parallelism } \\
\text { Positive feedback helps the quick } \\
\text { discovery of good solutions } \\
\text { Efficient in finding short paths } \\
\text { Distances changes adaptability } \\
\text { Convergence guaranteed }\end{array}$ & $\begin{array}{l}\text { Complex theoretical analysis } \\
\text { Random sequence decisions (Non- } \\
\text { independence) } \\
\text { Probability distribution changes by each } \\
\text { iteration } \\
\text { The search is experimental instead of } \\
\text { theoretical } \\
\text { The time convergence is uncertain }\end{array}$ & $\begin{array}{c}{[29],[32],} \\
{[46],[49],[50]}\end{array}$ \\
\hline $\mathrm{CS}$ & $\begin{array}{l}\text { Just one adjustment parameter } \\
\text { different from the population } \\
\text { number } \\
\text { Easy implementation } \\
\text { Ability to handle multi-criteria } \\
\text { optimization problems } \\
\text { Seeks to accelerate convergence }\end{array}$ & $\begin{array}{l}\text { If the values of probability to discovery an } \\
\text { invasive egg and the scale of the problem } \\
\text { of interest: } \\
\text { The Algorithm performance could be poor } \\
\text { and the iteration number increases } \\
\text { The convergence speed could be high but } \\
\text { cannot find best solutions }\end{array}$ & [47], [51] \\
\hline PSO & $\begin{array}{l}\text { The disruption is low to the } \\
\text { scalability of design variables } \\
\text { Easy parallelism for concurrent } \\
\text { processing } \\
\text { Not derivable } \\
\text { Quite efficient for the global search }\end{array}$ & $\begin{array}{l}\text { Trend to fast and premature convergence at } \\
\text { optimization midpoint } \\
\text { Slow convergence in the refined search } \\
\text { stage } \\
\text { Weak local search }\end{array}$ & $\begin{array}{l}{[27],[38]} \\
{[48],[52]}\end{array}$ \\
\hline GA & $\begin{array}{l}\text { Multi-objective optimization } \\
\text { Dynamic configuration based on } \\
\text { environment changes }\end{array}$ & $\begin{array}{l}\text { Requires prior knowledge of the system } \\
\text { High complexity with robust problems }\end{array}$ & [30], [53], [54] \\
\hline
\end{tabular}

\section{CHALLENGES OF RESEARCH}

The CRN has challenges to approach, and solutions to improve the QoS. It will ensure the spectrum managed in the best way. Then, we will expose some of these difficulties and how EAs can have a more significant impact.

Since PU can access the spectrum at any time, SU must be sensing the spectrum at all times and looking for new opportunities of available channels. Technologies have advanced and have allowed mobile terminals can sense the wireless spectrum. Because the sensing assignment is determined by some aspects such as energy, location, and costs of mobile terminals. It should be advised methods to improve the sensing spectrum.

In wireless sensor networks, unlicensed sensor nodes are used temporarily and idle licensed channels are occupied. New challenges have been identified in wireless sensor networks for detection and exchange in the cooperative spectrum. Problems such as adaptation in self-organization, fault tolerance and scalability in the network decrease the performance in the system.

When the number of unlicensed users increases in secondary networks, communication performance decreases and causes problems in selection and exchange of information. In order to solve the disadvantages of signal-to-noise interference, solutions must be proposed that dynamically manage the selection in the retransmission and spectrum allocation. EAs can be presented as a solution in the optimization of this problem. They canfind new routes given the restrictions presented by each network.

Currently, technology has been working on processes that reduce the delays in spectrum mobility and increase the total service time. For example, if optimization methods are used in the selection of the control channel, time will be reduced in the handoffs and the service time will be increased. EAs can propose solutions in this aspect by implementing optimization mechanics in the selection of the perfect control channel.

Another current challenge is focused on maximizing throughput capacity which presents a nonconvex [55]. Therefore, reformulation solutions have been proposed which will increase the mathematical cost and thus a delay in the service. This can represent a clear opportunity for EAs to present a contribution and propose a solution that does not involve reformulations in the problem [56]-[58]. For example, a genetic algorithm generates multiple solutions. 


\section{CONCLUSION}

Cognitive radio networks present a theoretical solution to the problem of inefficient use of the spectrum. This article presents an overview of new developments carried out by evolutionary algorithms that held cognitive radio network technologies. The art state has shown aspects such as service quality and energy costs can be improved by implementing cognitive radio technologies.

In Conclusion, this paper describes the capabilities and opportunities of evolutionary algorithms, as well as the current research challenges, in which there may be different points of view in applications for cognitive radios. It is important to highlight that although the literature presents solutions for the problems that have arisen in the sensing, decision, sharing, and mobility of the spectrum, there are still problems of optimization, learning, and classification in which evolutionary algorithms can offer solutions.

\section{ACKNOWLEDGEMENTS}

The authors of this article wish to acknowledge Colciencias and the Universidad Distrital Francisco José de Caldas for funding resources to develop this research.

\section{REFERENCES}

[1] E.U. Ogbodo, D. Dorrell, and A.M. Abu-Mahfouz, "Cognitive Radio based Sensor Network in Smart Grid: Architectures, Applications and Communication Technologies", IEEE Access, vol. 5, pp. 1-1, 2017.

[2] S.J. Shellhammer, A.K. Sadek, and W.Z.W. Zhang, "Technical Challenges for Cognitive Radio in the TV White Space Spectrum”, 2009 Inf. Theory Appl. Work., pp. 323-333, 2009.

[3] J. Wang and X. Zhang, "Statistical QoS-driven cooperative power allocation game over wireless cognitive radio networks", IEEE Wirel. Commun. Netw. Conf. WCNC, 2017.

[4] A.A. Khan, M.H. Rehmani, and A. Rachedi, "When Cognitive Radio meets the Internet of Things?", 2016 Int. Wirel. Commun. Mob. Comput. Conf. IWCMC 2016, pp. 469-474, 2016.

[5] S.P.T. Force., "ET Docket No. 02- 135 November 2002", 2002.

[6] A. Al-Tahmeesschi, M. López-Benítez, J. Lehtomäki, and K. Umebayashi, "Investigating the Estimation of Primary Occupancy Patterns under Imperfect Spectrum Sensing”, 2017 IEEE Wirel. Commun. Netw. Conf. Work. WCNCW 2017, 2017.

[7] E. Telatar, "Capacity of Multi-antenna Gaussian Channels", Trans. Emerg. Telecommun. Technol. 10, vol. 6, no. Nov, pp. 585-595, 1995.

[8] I. Lee, A.M. Chan, and C.E.W. Sundberg, "Space-Time Bit-Interleaved Coded Modulation for OFDM Systems", IEEE Trans. Signal Process., vol. 52, no. 3, pp. 820-825, 2004.

[9] M. López-benítez and F. Casadevall, "Spectrum occupancy in realistic scenarios and duty cycle model for cognitive radio", Adv. Electron. Telecommun., vol. 1, no. 1, pp. 26-34, 2010.

[10] R. Chakravarthy, Kaiyu Huang, Lin Zhang, and Z. Wu, "Primary User authentication of cognitive radio network using underlay waveform”, 2017 Cogn. Commun. Aerosp. Appl. Work., pp. 1-5, 2017.

[11] M. a McHenry, D. McCLOSKEY, D. Roberson, and J. MacDonald, "Spectrum occupancy measurements: Chicago, illinois, november 16-18, 2005”, Shar. Spectr. Company, Tech. Rep, pp. 2005-2011, 2005.

[12] S. Haykin, "Cognitive Radio: Brain-Empowered", IEEE J. Sel. AREAS Commun., vol. 23, no. 2, pp. 201-220, 2005.

[13] I.F. Akyildiz, W.Y. Lee, M.C. Vuran, and S. Mohanty, "NeXt generation/dynamic spectrum access/cognitive radio wireless networks: A survey", Comput. Networks, vol. 50, no. 13, pp. 2127-2159, 2006.

[14] L. Qiu, S. Liu, Y. Zhang, Y. Zhu, K. Tang, and Y. Zheng, "A 0.9-2.6 GHz Cognitive Radio Receiver with Spread Spectrum Frequency Synthesizer for Spectrum Sensing”, IEEE Sens. J., vol. 1748, no. c, pp. 1-1, 2017.

[15] D.E. Goldberg and J.H. Holland, "Genetic Algorithms and Machine Learning", Mach. Learn., vol. 3, no. 2, pp. 95-99, 1988.

[16] M. Bkassiny, Y. Li, and S.K. Jayaweera, "A survey on machine-learning techniques in cognitive radios", IEEE Commun. Surv. Tutorials, vol. 15, no. 3, pp. 1136-1159, 2013.

[17] A. He, S. Member, K.K. Bae, T.R. Newman, J. Gaeddert, K. Kim, R. Menon, L. Morales-tirado, J.J. Neel, Y. Zhao, J.H. Reed, W.H. Tranter, and L. Fellow, "A Survey of Artificial Intelligence", IEEE Trans. Veh. Technol., vol. 59, no. 4, pp. 1578-1592, 2010.

[18] M. Črepinšek, S.H. Liu, and M. Mernik, "Exploration and exploitation in evolutionary algorithms", ACM Comput. Surv., vol. 45, no. 3, pp. 1-33, 2013.

[19] N. Abbas, Y. Nasser, and K. El Ahmad, "Recent advances on artificial intelligence and learning techniques in cognitive radio networks", EURASIP J. Wirel. Commun. Netw., vol. 2015, no. 1, p. 174, 2015.

[20] U. Mehboob, J. Qadir, S. Ali, and A. Vasilakos, "Genetic algorithms in wireless networking: techniques, applications, and issues”, Soft Comput., vol. 20, no. 6, pp. 2467-2501, 2016.

[21] I.F. Akyildiz, W. Lee, M.C. Vuran, and S. Mohanty, "A Survey on Spectrum Management in Cognitive Radio Networks", IEEE Commun. Mag., vol. 46, no. 4, pp. 40-48, 2008.

[22] A. Fallis, "Notice of Proposed rule making and order", J. Chem. Inf. Model., vol. 53, no. 9, pp. 1689-1699, 2013.

[23] J. Elhachimi and Z. Guennoun, "An Artificial Intelligence Approach for Parking Facilities Management", Int. J. 
Comput. Technol. Appl., vol. 5, no. June, pp. 1219-1225, 2003.

[24] F.K. Jondral, "Software-Defined Radio Basics and Evolution to Cognitive Radio", EURASIP J. Wirel. Commun. Netw., vol. 2005, no. 3, p. 652784, 2005.

[25] H. Li, X. Cheng, K. Li, X. Xing, and T. Jing, "Utility-based cooperative spectrum sensing scheduling in cognitive radio networks", Proc. - IEEE INFOCOM, vol. 66, no. 1, pp. 165-169, 2013.

[26] M. Li, Y. Hei, and Z. Qiu, "Optimization of Non-convex Cooperative Spectrum Sensing with Modified Artificial Bee Colony Algorithm”, Proc. - 2014 IEEE Int. Conf. Comput. Inf. Technol. CIT 2014, pp. 70-75, 2014.

[27] X. Li and L. Liu, "Cooperative spectrum sensing for cognitive radios based on a PA-GABC algorithm", 2011 Int. Conf. Electron. Commun. Control, no. 2, pp. 2604-2607, 2011.

[28] L.I. Xinbin, S.H.I. Aiwu, and L.I.U. Lei, "Cognitive Radio Power Allocation Based on Artificial Bee Colony Algorithm”, Proc. 31st Chinese Control Conf., pp. 5809-5813, 2012.

[29] M. Bandyopadhyay and P. Bhaumik, "Zone based ant colony routing in mobile ad-hoc network", 2010 Second Int. Conf. Commun. Syst. NETworks (COMSNETS 2010), no. Ccc, pp. 1-10, 2010.

[30] F. Li, D. Zhu, F. Tian, and H. Li, "Cognitive Radio Spectrum Sharing using Improved Quantum Genetic Algorithm”, 2011 Int. Conf. Wirel. Commun. Signal, pp. 1-6, 2011.

[31] M.E. Bayrakdar and A. Calhan, "Optimization of spectrum handoff with artificial bee colony algorithm", 201725 th Signal Process. Commun. Appl. Conf., pp. 1-4, 2017.

[32] B. Preveze and A. Safak, "Effects of ant colony and fastest path routing algorithms on performance improvement of novel cognitive methods", Proc. - 6th Int. Conf. Wirel. Mob. Commun. ICWMC 2010, pp. 478-483, 2010.

[33] J. Qadir, "Artificial Intelligence Based Cognitive Routing for Cognitive Radio Networks", Artif. Intell. Rev., no. 1, pp. 25-96, 2013.

[34] D. Karaboga, “An idea based on honey bee swarm for numerical optimization”, Kayseri - Türkiye, 2005.

[35] T. Seeley, The Wisdom of the Hive. 1995.

[36] A. Colorni, M. Dorigo, and V. Maniezzo, "Distributed Optimization by Ant Colonies", Proc. ECAL91 - Eur. Conf. Artif. Life, Paris - Fr., no. or D, pp. 134-142, 1991.

[37] M Dorigo, V. Maniezzo, and A. Colorni, "The Ant System: An Autocatalytic Optimizing Process”, Milano, Italy, 1991.

[38] Z. Zhao, Z. Peng, S. Zheng, and J. Shang, "Cognitive radio spectrum allocation using evolutionary algorithms", IEEE Trans. Wirel. Commun., vol. 8, no. 9, pp. 4421-4425, 2009.

[39] R.B. Payne and M.D. Sorenson, The Cuckoos, 1st ed. New York: Oxford University Press, 2005.

[40] X.S. Yang and S. Deb, "Cuckoo Search via Lévy Flights", World Congr. Nat. Biol. Inspired Comput. 2009. NaBIC 2009., pp. 210-214, 2010.

[41] J. Kennedy and R.C. Eberhart, "Particle Swarm Optimization", Proc. IEEE Int. Conf. Neural Networks IV, pages, vol. 4, pp. 1942-1948, 1995.

[42] C.W. Reynolds, "Flocks, herds and schools: A distributed behavioral model", ACM SIGGRAPH Comput. Graph., vol. 21, no. 4, pp. 25-34, 1987.

[43] S. Ashrafinia, U. Pareek, M. Naeem, and D.C. Lee, "Binary Artificial Bee Colony for Cooperative Relay Communication in Cognitive Radio Systems", 2012 IEEE Int. Conf. Commun., pp. 1550-1554, 2012.

[44] A. Ghasemi, F. Qassemi, M. Biguesh, and M.A. Masnadi-Shirazi, "Channel assignment based on bee algorithms in multi-hop cognitive radio networks", IET Commun., vol. 8, no. 13, pp. 2356-2365, 2014.

[45] C. Peng, H. Zheng, and B.Y. Zhao, "Utilization and Fairness in Spectrum Assignemnt for Opportunistic Spectrum Access", Mob. Networks Appl., vol. 11, no. 4, pp. 555-576, 2006.

[46] H. Ohize and M. Dlodlo, "Ant Colony System Based Control Channel Selection Scheme for Guaranteed Rendezvous in Cognitive Radio Ad-hoc Network", 27th Annu. IEEE Int. Symp. Pers. Indoor Mob. Radio Commun. PIMRC, 2016.

[47] W. Yuan, M. Yang, Q. Guo, X. Wang, and X. Feng, "Improved Cuckoo Search Algorithm for Spectrum Sensing in Sparse Satellite Cognitive Systems", IEEE 84th Veh. Technol. Conf., pp. 1-5, 2016.

[48] A. Alhammadi, M. Roslee, and M.Y. Alias, "Analysis of Spectrum Handoff Schemes in Cognitive Radio Network Using Particle Swarm Optimization”, IEEE 3rd Int. Symp. Telecommun. Technol. (ISTT), Kuala Lumpur, pp. 103-107, 2016.

[49] Q. He and P. Zhang, "Dynamic Channel Assignment Using Ant Colony Optimization for Cognitive Radio Networks", Veh. Technol. Conf. (VTC Fall), 2012 IEEE, pp. 1-5, 2012.

[50] Z. Zhu, J. Chen, and S. Zhang, "Spectrum Allocation Algorithm Based on Improved Ant Colony in Cognitive Radio Networks", 2016 IEEE Int. Conf. Internet Things IEEE Green Comput. Commun. IEEE Cyber, Phys. Soc. Comput. IEEE Smart Data, pp. 376-379, 2016.

[51] M. Grissa, A.A. Yavuz, and B. Hamdaoui, "Cuckoo Filter-Based Location-Privacy Preservation in DatabaseDriven Cognitive Radio Networks”, 2015 World Symp. Comput. Networks Inf. Secur., 2015.

[52] L. Zhai and H. Wang, "Crowdsensing Task Assignment Based on Particle Swarm Optimization in Cognitive Radio Networks", Hindawi, Wirel. Commun. Mob. Comput., 2017.

[53] R. Huang, J. Chang, Y. Ren, F. He, and C. Guan, "Spectrum Allocation of Cognitive Radio Network Based on Optimized Genetic Algorithm in Underlay Network Rixing”, 2016 8th IEEE Int. Conf. Commun. Softw. Networks, pp. 418-422, 2016.

[54] J. Elhachmi and Z. Guennoun, "Evolutionary Algorithm for Dynamic Spectrum Allocation in Cognitive Radio System", 2016 4th IEEE Int. Colloq. Inf. Sci. Technol., pp. 702-706, 2016.

[55] M. Saeedzarandi, "Optimization of Multiband Sensing -Time-Adaptive Detection in Cognitive Radio Networks Using Artificial Inmmune Algorithm”, 2nd Conf. Swarm Intell. Evol. Comput., vol. 0, no. 1, pp. 29-34, 2017. 
[56] J. Avila and K. Thenmozhi, "Multiband OFDM for Cognitive Radio - A Way for Cyclostationary Detection and Interference Cancellation", International Journal of Electrical and Computer Engineering, vol. 6, no. 4, pp. 1702-1709, 2016.

[57] C.S. Preetham, M.S.G. Prasad, D.S.S.L. Saranya, Charan, D. Bhargava and V. Rohit, "Performance Analysis of Cooperative Hybrid Cognitive Radio Network with Various Diversity Techniques", International Journal of Electrical and Computer Engineering, vol. 6, no. 5, pp. 2125-2133, 2016.

[58] R. Shahbazian and S. Ali Ghorashi, "Localization of Distributed Wireless Sensor Networks using Two Sage SDP Optimization", International Journal of Electrical and Computer Engineering, vol. 7, no. 3, pp. 1255-1261, 2017.

\section{BIOGRAPHIES OF AUTHORS}

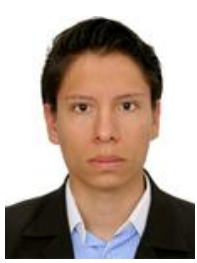

Miguel Tuberquia, Electronic Engineer from the District University Francisco Jose de Caldas in Bogota-Colombia. Interested in Artificial Intelligence, control, mechanical design, embedded systems and Cognitive Radio. Currently working as a research in ARMOS researching group and Colciencias-Colombia. He has been developing a new algorithm for optimizing the spectrum allocation in Colombia.

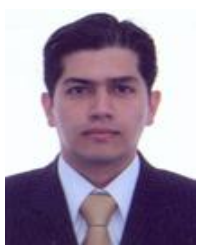

Cesar Hernandez, He received his BS degree in Electronic Engineer, and his MS degree in Information Sciences and Communications from the Universidad Distrital Francisco José de Caldas, Colombia, in 2003 and 2007, respectively. He received his PhD in Computer Systems Engineering from the Universidad Nacional de Colombia in 2017. He has publications in telecommunications area in international journals, books, and conferences. His current research interests are in dynamic spectrum access and microcontrollers. PhD Hernandez is Titular Professor at the Universidad Distrital Francisco José de Caldas and director of SIREC research group. 